\title{
Capacidad de absorción: revisión de la literatura y un modelo de sus determinantes
}

\section{Absorptive capacity: a literature review and a model of its determinants}

Dr. Gerardo J. Zapata Rotundo es profesor/investigador titular del Decanato de Ciencias Económicas y Empresariales de la Universidad Centroccidental Lisandro Alvarado (Venezuela) (zapager@yahoo.com) (https://orcid.org/0000-0001-7021-5443).

Dra. Aymara Hernández Arias es profesora/investigadora titular del Decanato de Ciencias Económicas y Empresariales de la Universidad Centroccidental Lisandro Alvarado (Venezuela) (aymarah@yahoo.com) (https://orcid.org/0000-0002-1040-4709).

\section{Resumen}

El presente artículo tiene como objetivo llevar a cabo una revisión de la literatura sobre uno de los constructos más relevantes en el estudio del comportamiento de las organizaciones que surge inicialmente a partir del trabajo de Cohen \& Levinthal (1990). El mismo se refiere a la capacidad de absorción que ha tenido una amplia aplicación en distintos campos de estudios de la organización, tales como: aprendizaje y gestión del conocimiento, procesos de innovación y desarrollo tecnológico, administración estratégica, dirección de recursos humanos, diseño organizativo, entre otros. La investigación, abordada bajo un enfoque metodológico de tipo descriptivo y con diseño documental, pretende avanzar en la integración y comprensión de este constructo a través de la identificación y discusión de diferentes aspectos referidos a su origen, componentes, determinantes, su relación con la cognición del individuo y la importancia que tiene para el desarrollo, el desempeño y el logro de ventajas competitivas, proporcionando así una visión general para su gestión y aplicación. Como resultado de la investigación se ofrece un modelo de los factores que definen la capacidad de absorción de la organización, alrededor de tres niveles principales: organizacional, del entorno y cognitivo, destacándose este último como un factor diferenciador de los modelos que tradicionalmente han sido desarrollados por la literatura.

\begin{abstract}
The present article aims to carry out a review of the literature on one of the most relevant constructs in the study of organizational behavior that emerged from the work of Cohen \& Levinthal (1990). We refer to the absorptive capacity, which has had a wide attention and application in different fields: learning and knowledge management, innovation processes and technological development, strategic management, human resource management, organizational design, among others. In this way, the research is approached according to the type of descriptive study, with documentary design. Therefore, we intend to advance in the integration and comprehension of the capacity of absorption through the identification and discussion of different aspects related to its origin, components, determinants, its relation with the cognition of the individual and the importance that it has for the development, performance and the achievement of competitive advantages on the company. This provides a general vision for its management and application, and a model of its determinants is proposed. The factors that define the construct are presented through a model, among them: organizational, environment and cognitive. The latter is highlighted as a differentiating factor from the models that have traditionally been developed by literature.
\end{abstract}

\section{Palabras claves I keywords}

Capacidad de absorción, cognición, conocimientos, entorno, organización, ventajas competitivas. Absorptive capacity, environment, competitive advantages, cognition, organization, knowledge.

Cómo citar: Zapata Rotundo, G.J. y Hernández Arias, A. (2018). Capacidad de absorción: revisión de la literatura y un modelo de sus determinantes. Retos Revista de Ciencias de la Administración y Economía, 8(16), 121-140. https://doi.org/10.17163/ret.n16.2018.09 


\section{Introducción}

La teoría de la organización proporciona herramientas conceptuales y empíricas que contribuyen al estudio, análisis y comprensión del comportamiento de las organizaciones (Zapata \& Mirabal, 2018), ayudando así «a explicar una parte de la realidad organizacional, con el fin de dar un mayor entendimiento sobre su adecuada operación» (Sánchez y Nélida, 2017, p. 66). En ese sentido, y de manera específica, el concepto de capacidad de absorción (CA) ha sido un tema de especial atención para entender los diversos fenómenos organizacionales (Gao et al., 2017), fundamentalmente aquellos vinculados con los procesos de gestión del conocimiento, aprendizaje, innovación y desempeño.

Al respecto, Lichtenthaler (2016a, p. 1) señala que la literatura «subraya la importancia que juega la capacidad de absorción en el desarrollo del conocimiento, la promoción de la innovación abierta, la gestión de alianzas estratégicas, en el desarrollo del aprendizaje, la creación de variedad estratégica y en la mejora del rendimiento financiero». Por su parte, King \& Lakhani (2011) indican que los cambios frecuentes en el contexto de la innovación han hecho más importante el estudio de la CA, constructo que fue introducido por Cohen \& Levinthal (1990) como condición para lograr una alta competencia innovadora (Duchek, 2013, 2015), que hace posible que el conocimiento externo disponible en el entorno se haga utilizable dentro de la organización (Schweisfurth \& Raasch, 2018), convirtiéndose, por tanto, «en un fuerte predictor de innovación y transferencia de conocimientos» (Zou et al., 2018, p. 1).

La capacidad de absorción es la habilidad de la organización para identificar el valor del conocimiento útil ubicado en su entorno, asimilarlo, transformarlo e integrarlo a su base de conocimientos, y así aplicarlo a través de los procesos y acciones relacionados con la innovación, la inversión I+D y la competitividad (Cohen \& Levinthal, 1990). De este modo, su desarrollo y mantenimiento se considera de vital importancia para la supervivencia y el éxito de la empresa, puesto que contribuye a mejorar, organizar y dirigir los conocimientos hacia el logro de sus metas y objetivos (Zahra \& George, 2002). Asimismo, la inversión en CA permite incrementar la habilidad de la empresa para anticiparse a las tendencias del mercado (Cohen \& Levinthal, 1994). Todo lo anterior se traduce en ventajas competitivas, contribuyendo así al planteamiento de que el proceso de innovación tecnológica requiere de la integración del conocimiento interno y externo con la competencia inventiva y creadora de la organización (King \& Lakhani, 2011).

En su trabajo seminal, Cohen y Levinthal (1990) manifiestan que se requiere de más investigación para entender los procesos de decisión que determinan las inversiones de las organizaciones en CA. De este modo, desde la aparición de su artículo se han desarrollado trabajos orientados a consolidar la teoría de la CA como una palanca de apoyo para los investigadores y académicos dedicados a comprender y explicar el desarrollo y evaluación de la organización. Investigaciones en diferentes áreas relacionadas con temas organizacionales así lo confirman (ver tabla 1). 
Tabla 1. Investigaciones en temas organizacionales y capacidad de absorción

\begin{tabular}{|l|l|}
\hline \multicolumn{1}{|c|}{ Área } & \multicolumn{1}{c|}{ Autores } \\
\hline Gestión del conocimiento & $\begin{array}{l}\text { Van den Bosch et al. (1999), Minbaeva et al. (2003), } \\
\text { Camisón \& Forés (2014), Mariano \& Walter (2015), } \\
\text { Valentim et al. (2016), Qian \& Jung (2017). }\end{array}$ \\
\hline Procesos de innovación & $\begin{array}{l}\text { Volberda et al. (2010), Kostopoulos et al. (2011), } \\
\text { King \& Lakhani (2011), Chen \& Chang (2012), } \\
\text { Cassol et al. (2016), Kang \& Lee (2017), Arias et al., } \\
\text { (2017), Saiz et al. (2018), Schweisfurth \& Raasch } \\
\text { (2018), Zou et al. (2018). }\end{array}$ \\
\hline Alianzas estratégicas & Hoang y Rothaermel (2005). \\
\hline Estudio de filiales extranjeras & Minbaeva et al. (2003, 2014) y Peltokorpi (2017). \\
\hline $\begin{array}{l}\text { Flujo de conocimientos entre } \\
\text { regiones }\end{array}$ & $\begin{array}{l}\text { Schillaci et al. (2013), Miguélez \& Moreno (2015), } \\
\text { Juknevičiené (2017). }\end{array}$ \\
\hline Desempeño organizacional & $\begin{array}{l}\text { Lane et al. (2001), Kostopoulos et al. (2011), Mara- } \\
\text { belli \& Newell (2014), Cepeda et al. (2016), Lichten- } \\
\text { thaler (2016a), Guisao et al. (2017), Zou et al. (2018). }\end{array}$ \\
\hline $\begin{array}{l}\text { Sistemas y tecnología de infor- } \\
\text { mación }\end{array}$ & $\begin{array}{l}\text { Roberts et al. (2012), Gao et al. (2017), Guisao et al. } \\
\text { (2017). }\end{array}$ \\
\hline Aprendizaje organizacional & $\begin{array}{l}\text { Lane \& Lubatkin(1998), Lane et al. (2001), Lane et } \\
\text { al. (2006), Cepeda et al. (2016), Vargas \& Muratalla } \\
\text { (2017). }\end{array}$ \\
\hline $\begin{array}{l}\text { Contratación y movilidad la- } \\
\text { boral }\end{array}$ & Leiva et al. (2017). \\
\hline $\begin{array}{l}\text { Empresas familiares e innova- } \\
\text { ción }\end{array}$ & Charão \& Matos (2017). \\
\hline
\end{tabular}

De este modo, la presente investigación tiene como objetivo llevar a cabo una revisión de la literatura alrededor del constructo capacidad de absorción, a fin de discutir aspectos relativos a su origen, significado, componentes, niveles de análisis, relación con la cognición del individuo y la importancia que tiene para el desarrollo, desempeño y obtención de ventajas competitivas para la organización. Finalmente, se propone un modelo de sus factores determinantes.

Desde el punto de vista metodológico, esta investigación se abordó bajo un enfoque descriptivo y con diseño documental. El protocolo de selección de las referencias bibliográficas se inició con la definición de las variables bajo estudio, en este caso la capacidad de absorción como uno de los componentes fundamentales de las capacidades dinámicas de la organización. Se hizo la lectura sistemática de la teoría fundamental y del estado de la cuestión del tema estudiado, así como la selección de criterios de búsqueda a fin de ubicar materiales relevantes en el área administrativo-gerencial de acuerdo al objetivo planteado. Este proceso se llevó a cabo a través de la revisión de bases de datos especializadas tales como InfoTrac, EBSCOHost y ProQuest, que ofrecen artículos a texto completo de revistas científicas arbitradas e indexadas las cuales garantizan la revisión por pares en cuanto a la coherencia del contenido y la aplicación 
rigurosa de los lineamientos metodológicos. Ello dio como resultado emergente 75 artículos científicos de carácter teórico y empírico, los cuales fueron analizados de forma crítica (Viniegra, 2001; Garcés \& Duque, 2007 y Díaz, 2012). Adicionalmente, se seleccionaron 5 libros especializados, todo con el propósito de integrar y fundamentar los planteamientos realizados en la propuesta presentada sobre los factores determinantes de la capacidad de absorción de las organizaciones.

\section{Origen y definición de la capacidad de absorción (CA)}

La CA tiene su origen en los trabajos seminales de Cohen \& Levinthal $(1989,1990)$ quienes la definen como «la habilidad de la empresa para identificar, asimilar y explotar el conocimiento del entorno» (Cohen \& Levinthal, 1989, p. 569). En un trabajo posterior, señalan que es la habilidad de la empresa para reconocer el valor de la nueva información, asimilarla y aplicarla para fines comerciales y en los procesos de innovación (Cohen \& Levinthal, 1990).

Cohen \& Levinthal $(1989,1990)$ indican que una de las capacidades primordiales de la empresa son las actividades de investigación y desarrollo que generan conocimiento útil necesario para su buen desempeño. Esa capacidad se aprecia como crucial en las condiciones del entorno actual (Camisón \& Forés, 2014) el cual se caracteriza por ser complejo, cambiante y competitivo.

Se desprende de Cohen \& Levinthal (1990) la importancia de las motivaciones y las habilidades individuales como factores clave de la CA (Minbaeva et al. 2003, 2014). $\mathrm{Al}$ respecto, señalan que «nuestra perspectiva implica que la facilidad de aprendizaje, y por tanto la adopción de tecnología, se ve afectada por el grado que una determinada innovación está relacionada con la base de conocimientos pre-existentes de sus posibles usuarios» (Cohen \& Levinthal, 1990, p. 148), por ejemplo «los computadores personales se difundieron más rápidamente al principio entre los consumidores y las empresas que tenían experiencia previa en mainframes o minicomputadoras» (Cohen \& Levinthal, 1990, p. 149). De esta forma, consideran que los individuos aprenden acumulando conocimiento a lo largo de su actuación dentro de la organización. En esencia, la CA gira alrededor de la idea de que la experiencia acumulada por adopción e invención mejora la capacidad de reconocer y absorber nuevos conocimientos de alta calidad, así como de crear nuevas y valiosas invenciones (King \& Lakhani, 2011); es, por tanto, una actividad recursiva.

Cohen \& Levinthal (1990) también consideran que la organización posee memoria que facilita el almacenaje, recuperación y aplicación del conocimiento asociado tanto a nivel individual como organizacional, y que motiva, a su vez, el proceso de aprendizaje, dándoles una vital importancia al modelo cognitivo del individuo. Sin embargo, aclaran que no solo se refiere a la sumatoria de capacidades de absorción de sus empleados, sino también implica aspectos organizativos que incluyen, por ejemplo, la cultura organizacional, la inversión en $\mathrm{I}+\mathrm{D}$, las prácticas y políticas de recursos humanos y la estructura organizativa, con lo cual es un producto de una serie de relaciones con el entorno, la estrategia, la estructura, los procesos y la cultura organizacional, así como también de las motivaciones y habilidades individuales para absorber nuevos conocimientos (Lane et al., 2006). En este sentido, existen tres niveles de análisis 
de la capacidad de absorción: individual, grupal y organizacional, que se relacionan y coexisten en la organización.

La capacidad de absorción «juega un papel clave en la innovación y en el desempeño, independientemente del tamaño de las empresas y del nivel de recursos» (Valentim et al., 2016, p. 4), es crucial para el proceso de creación de conocimiento empresarial (Charão \& Matos, 2017), y desempeña un rol primordial para que las capacidades tecnológicas de información de la empresa influyan sobre sus resultados (Guisao et al., 2017). Así, las empresas con altos niveles de CA pueden gestionar el conocimiento externo más eficientemente (Escribano et al., 2009). Debido a esto, es crítica para cualquier empresa que busca alcanzar nuevas formas de ventajas competitivas, desempeños superiores y resultados innovadores en los bienes y servicios que ofrece según las exigencias del entorno cambiante. Cabe resaltar que la CA se fundamenta en el hecho de que la organización no tiene el conocimiento interno suficiente para mejorar o desarrollar todos sus procesos de innovación (Aguilar-Olaves et al., 2014; Duchek, 2015).

Esas ideas, por tanto, permiten ubicar a la CA dentro del ámbito de dos enfoques identificados en la teoría de la organización: la visión basada en el conocimiento, que es una de las derivaciones de la teoría de recursos y capacidades; y dentro del contexto de las teorías de control externo ${ }^{1}$ de la organización, específicamente en la teoría dependencia de recursos propuesta por Pfeffer \& Salancik (1978). Esta última, plantea que la organización no posee las capacidades, ni es autosuficiencia, para producir y controlar todos los recursos y servicios que necesita para alcanzar un funcionamiento adecuado, por lo que debe recurrir a las relaciones de intercambio e interdependencia con otras organizaciones para obtenerlos. Dentro de esos recursos se encuentra el conocimiento externo necesario a los fines de cumplir con los distintos procesos organizacionales.

A manera de síntesis, en la tabla 2 se presentan algunas definiciones de capacidad de absorción.

Tabla 2. Definiciones de Capacidad de Absorción (CA)

\begin{tabular}{|l|l|}
\hline \multicolumn{1}{|c|}{ Autores } & \multicolumn{1}{c|}{ Definición } \\
\hline $\begin{array}{l}\text { Lane \& Lubat- } \\
\text { kin (1998) }\end{array}$ & $\begin{array}{l}\text { Habilidad de la empresa para asimilar y aplicar el conocimiento } \\
\text { proveniente de otra empresa. Esa capacidad depende de: (1) la si- } \\
\text { militud entre las empresas; (2) el conocimiento base; (3) la estruc- } \\
\text { tura organizativa; y (4) la lógica dominante. Este último se ubica } \\
\text { dentro del marco de la psicología cognitiva. }\end{array}$ \\
\hline $\begin{array}{l}\text { Lane et al. } \\
(2006)\end{array}$ & $\begin{array}{l}\text { La empresa utiliza el conocimiento del entorno a través de tres } \\
\text { procesos secuenciales: (a) el reconocimiento y entendimiento de } \\
\text { nuevo conocimiento mediante el aprendizaje exploratorio; (b) la } \\
\text { asimilación de nuevos conocimientos por medio del aprendizaje } \\
\text { transformador; y (c) el uso del conocimiento asimilado para gene- } \\
\text { rar otros nuevos mediante el aprendizaje. }\end{array}$ \\
\hline
\end{tabular}

1 En el enfoque de control externo se ubican las teorías: contingente, dependencia de recursos, institucional y ecología de las poblaciones. Forman también parte del enfoque de adaptación. 


\begin{tabular}{|l|l|}
\hline \multicolumn{1}{|c|}{ Autores } & \multicolumn{1}{c|}{ Definición } \\
\hline $\begin{array}{l}\text { Todorova \& } \\
\text { Durisin (2007) }\end{array}$ & $\begin{array}{l}\text { Capacidad de una empresa para valorar, adquirir, asimilar o trans- } \\
\text { formar y explotar el conocimiento externo en los procesos organi- } \\
\text { zacionales. }\end{array}$ \\
\hline $\begin{array}{l}\text { Schillaci et al. } \\
(2013)\end{array}$ & $\begin{array}{l}\text { Extiende la capacidad de absorción a la competencia entre las re- } \\
\text { giones o territorios, y la definen como la capacidad de una región } \\
\text { para identificar, asimilar y explotar el conocimiento. }\end{array}$ \\
\hline $\begin{array}{l}\text { Chang et al. } \\
(2014)\end{array}$ & $\begin{array}{l}\text { Conjunto de habilidades de la empresa para adquirir, asimilar, } \\
\text { transformar y explotar el conocimiento para producir nuevas ca- } \\
\text { pacidades. }\end{array}$ \\
\hline $\begin{array}{l}\text { Garzón (2015) } \\
\text { ma habilidad de la organización para reconocer el valor del conoci- } \\
\text { to de la organización, y aplicarlo en el ámbito de sus operaciones. }\end{array}$ \\
\hline $\begin{array}{l}\text { Martinkenaite } \\
\text { \& Breunig } \\
(2016)\end{array}$ & $\begin{array}{l}\text { Conjunto de procesos secuenciales de aprendizaje exploratorio, } \\
\text { transformador y explotador en los que interactúan dinámicamen- } \\
\text { te las habilidades individuales y organizacionales. }\end{array}$ \\
\hline $\begin{array}{l}\text { Peltokorpi } \\
(2017)\end{array}$ & $\begin{array}{l}\text { Es la capacidad de las filiales, a través de sus miembros, de absor- } \\
\text { ber, asimilar y utilizar nueva información disponible en la empre- } \\
\text { sa multinacional. }\end{array}$ \\
\hline
\end{tabular}

\section{La capacidad de absorción como capacidad dinámica de la organización}

La teoría de capacidad dinámica se considera una ramificación de la teoría de recursos y capacidades (TRC). Ésta centra su análisis en la importancia de los recursos y capacidades internas de la organización como fuente principal de sus ventajas competitivas, prestándole poca atención a los factores del entorno donde se desenvuelve. Reivindica el rol que juegan las características internas -recursos y capacidades- como la clave para el establecimiento de las políticas y estrategias de la organización (Zapata \& Hernández, 2014; Zapata \& Mirabal, 2018). Sus inicios se ubican en los planteamientos de Penrose (1959), quien propone que el centro de competencias distintivas de la empresa lo constituyen los recursos -tangibles e intangibles- que posee, y las habilidades que utiliza para aprovecharlos de la mejor manera posible. Cuestiona esta teoría que el desarrollo de la empresa depende fundamentalmente de la dinámica del entorno, tal como lo plantean el enfoque de adaptación y de control externo.

Sin embargo, esta posición epistemológica ha tenido críticas que la consideran restrictiva y estática ( $v$. gr. González \& Hurtado, 2014) al no prestar atención, por una parte, a la dinámica existente entre organización y entorno (Zapata \& Mirabal, 2018), y por otra parte, al darle relevancia a la heterogeneidad y a las características de los recursos -valiosos, raros, no imitables y no equivalentes estratégicamente- como elementos estratégicos primordiales para la organización. Al respecto, Cruz et al. (2009, p. 111) plantea que «tales características no perdurarán en el tiempo ante los cambios que afectan a los mercados de factores y/o productos», o que en entornos que cambian rápidamente o mercados altamente globalizados, la TRC no tiene los argumentos sufi- 
cientes para explicar por qué las empresas con recursos semejantes no necesariamente alcanzan los mismos desempeños (Chang et al., 2014).

Surge entonces la teoría de capacidades dinámicas a finales de la década de los años noventa del siglo pasado, como una manera de dinamizar el enfoque de recursos y capacidades en respuesta a la necesidad de las organizaciones de adaptarse e innovarse de acuerdo a los requerimientos y dinámica del entorno (González \& Hurtado, 2014; Zapata \& Mirabal, 2018). De este modo, Teece et al. (1997) y Teece \& Leih (2016) establecen que las capacidades dinámicas contribuyen con la empresa a integrar, construir y reconfigurar los recursos y capacidades para dar respuestas rápidas a los entornos cambiantes, e implican actividades de más alto nivel que le permiten dirigir y mejorar sus capacidades ordinarias en la búsqueda de la producción de los bienes y servicios ajustados a las exigencias y circunstancias del entorno; y que ayuden, además, en el diseño e implementación de un modelo de negocio adaptado a esas circunstancias (Teece \& Linden, 2017).

Así, la CA se considera dentro de la literatura de las capacidades dinámicas como uno de sus componentes principales (Wang \& Ahmed, 2007; Valencia-Rodríguez, 2015; Garzón, 2015; Lin et al., 2016; Vargas \& Muratalla, 2017; Zapata \& Mirabal, 2018). Ya Cohen \& Levinthal (1990, p. 133) habían señalado en su trabajo seminal que la «capacidad de absorción no solo reside en un solo individuo, sino que depende del enlace de un mosaico de capacidades individuales». Es un constructo utilizado para explicar la complejidad de los fenómenos organizacionales a través de su participación como una capacidad dinámica que permite la transformación mediante la evolución o cambio en los procesos organizacionales y de innovación (Vargas \& Muratalla, 2017; Rodríguez \& Da Cunha, 2018). Es reconocida entonces la CA como una capacidad dinámica, puesto que a través de ella la empresa puede desarrollar habilidades de asimilación, aprendizaje, transferencia y explotación del conocimiento externo (González \& Hurtado, 2014; Vargas \& Muratalla, 2017), que contribuye a reconfigurar las capacidades ordinarias que posee (Roberts et al., 2012).

Al respecto, Zahra \& George (2002) sugieren que la adquisición, asimilación, transformación y explotación del conocimiento que se integran en el constructo CA poseen características de capacidades dinámicas, puesto que influyen en la empresa para crear y desplegar el conocimiento necesario para construir competencias organizacionales tales como en marketing, distribución y producción que hacen posible enfrentar los entornos turbulentos. De este modo, y siguiendo las ideas anteriores, Verona y Ravasi (2003), Lin et al. (2016) y Garzón (2015) muestran que la capacidad de las empresas para adquirir nuevos conocimientos, asimilarlos e integrarlos a la base de conocimientos ya existentes, son un factor determinante para generar e impulsar las capacidades dinámicas. En ese mismo orden de ideas, Zapata \& Mirabal (2018) argumentan que a través de la CA es posible que la organización desarrolle sus propias capacidades en los procesos de producción, organización y prestación de servicios. En resumen, mientras que una empresa demuestre mayor $\mathrm{CA}$, exhibirá más una mayor capacidad dinámica (Wang \& Ahmed, 2007), que se reconoce en la naturaleza y generación de ventajas competitivas (Zahra \& George, 2002). 


\section{Componentes de la capacidad de absorción}

De acuerdo al modelo Cohen \& Levinthal $(1989,1990)$ la capacidad de absorción tiene una naturaleza multidimensional que abarca tres capacidades: (a) reconocimiento del valor del nuevo conocimiento -explorar el conocimiento-; (b) asimilación de ese conocimiento que implica comprenderlo y adicionarlo a los procesos y rutinas de la organización -transformación del conocimiento-; y (c) aplicar esos conocimientos para la obtención de nuevos productos -explotarlo-. De igual forma, Aguilar-Olavés et al. (2014) explican que las empresas pasan por un proceso secuencial donde primero se reconoce el conocimiento potencialmente valioso (aprendizaje explorador), luego se asimila ese conocimiento (aprendizaje transformador), y finalmente se utiliza para innovar y crear nuevo conocimiento con valor de mercado (aprendizaje explotador).

A partir de la definición de los componentes de la CA en los trabajos de Cohen \& Levinthal $(1989,1990)$, se han formulado diversos modelos que coinciden en un punto común: es un generador de ventajas competitivas. A continuación, se describen algunos de los más relevantes.

Lane et al. (2001). Destacan el papel crítico de la CA durante el proceso de aprendizaje y desempeño interorganizacional. De este modo, proponen tres componentes en un entorno internacional de joint venture: capacidad de comprender el conocimiento externo, asimilarlo y aplicarlo. El primero, depende de la existencia de vínculos en los campos de acción de las empresas, a la similitud de sus problemas y a las prioridades que le asignan. Por su parte, la capacidad de asimilar el conocimiento externo está relacionada con la flexibilidad y la adaptabilidad que implican un alto nivel de entrenamiento del socio extranjero y de un aprendizaje previo que contribuyen con la reducción de la ambigüedad del nuevo conocimiento y su conexión con el existente. Finalmente, la aplicación del conocimiento externo implica la habilidad de difundirlo e integrarlo a las actividades de la organización.

Zahra \& George (2002). Bajo la idea de una reconceptualización de la CA, ofrecen un cambio al modelo original de tres dimensiones propuesto por Cohen \& Levinthal $(1989,1990)$. De este modo, la conceptualizan como «un conjunto de rutinas y procesos organizacionales por medio de los cuales le permiten a la empresa adquirir, asimilar, transformar y explotar el conocimiento externo. Esas cuatro capacidades organizativas se refuerzan entre sí para generar capacidad de absorción» (Zahra \& George, 2002, p. 186). Dichas rutinas, destacan los autores, proporcionan mecanismos estructurales, sistemáticos y procedimentales que contribuyen con la empresa a crear valor en sus productos y de esa manera lograr ventajas competitivas sostenibles.

La modificación de Zahra \& George (2002) al modelo de Cohen \& Levinthal (1990) gira en torno al cambio de tres a cuatro dimensiones: adquisición, asimilación, transformación y aplicación. (a) Adquisición, se identifica y se reconoce el valor del conocimiento externo que será seleccionado y luego utilizado en las operaciones de la empresa. (b) Asimilación, capacidad de la empresa para absorber y transferir el conocimiento externo. Se alcanza mediante el conjunto de rutinas y procesos necesarios para analizar, interpretar y comprender el conocimiento adquirido. (c) Transformación, habilidad de la empresa para desarrollar y mejorar los procesos y rutinas que facilitan la transferencia y combinación del conocimiento existente a partir de uno nuevo. Esto 
se logra, argumentan los autores, incorporando o eliminando conocimiento o interpretándolo de forma diferente. Y (d) aplicación o explotación, que «se basa en las rutinas que permiten a la empresa refinar, ampliar y aprovechar las competencias existentes o crear nuevas competencias incorporando el conocimiento adquirido y transformado a sus operaciones» (Zahra \& George, 2002, p. 190).

Ahora bien, Zahra \& George (2002, p. 191) con el argumento de que «la organización puede adquirir y asimilar conocimiento, pero no necesariamente tiene la capacidad de transformarlo y explotarlo para generar beneficios», agrupan entonces las cuatro dimensiones en dos categorías: «capacidad de absorción potencial» (PACAP), y «capacidad de absorción realizada» (RACAP). La PACAP, considera los procesos mediante los cuales la empresa puede absorber el conocimiento externo a través de su capacidad de adquisición y asimilación, pero no se garantiza de forma automática su transformación y aplicación (Kang \& Lee, 2017). Mientras que la RACAP incorpora los procesos que utilizan el conocimiento externo para los propósitos de la organización. Se logra esto a través de sus habilidades de transformación y explotación. Apoyando ese argumento, Cassol et al. (2016) explican que la PACAP es un instrumento para adquirir y asimilar el conocimiento pero que se encuentra en un nivel «relativamente adormecido» hasta que exista una verdadera razón para su uso, con lo cual se convierte en RACAP.

Todorova \& Durisin (2007). Plantean cuatro componentes: (a) reconocimiento del valor del conocimiento, retoman la idea inicial de Cohen \& Levinthal (1990); (b) adquisición; (c) transformación o asimilación; y (d) explotación. El reconocimiento del valor del conocimiento es considerado como un paso previo a su adquisición, cuestión que la ubican dentro del proceso de adquisición (Lane \& Lubatkin, 1998; Zahra \& George, 2002; Volberda et al., 2010; Duchek, 2013, 2015). Esa habilidad de reconocer el valor del nuevo conocimiento, es un componente importante de CA puesto que su valoración no es automática, está sesgada y necesita ser promovida para dar lugar a la absorción, por lo que «la capacidad de aprender, es decir, absorber el conocimiento externo, depende en gran medida de la capacidad de valorar adecuadamente el nuevo conocimiento» (Todorova \& Durisin, 2007, p. 777). Con respecto a la asimilación o transformación -esto último propuesto por Zahra \& George (2002)-, se señala que los conocimientos que intenta absorber la organización pueden avanzar y retroceder entre los procesos de asimilación y transformación antes de que sean incorporados con éxito en sus estructuras de conocimientos para su posterior explotación y aplicación.

Forés \& Camisón (2008) y Camisón \& Forés (2014). Proponen cuatro dimensiones de la CA diferenciadas pero complementarias: adquisición, asimilación, transformación y explotación. La adquisición la conceptualizan, partiendo de las ideas de Lane \& Lubatkin (1998) y Zahra \& George (2002), como «la habilidad de una empresa para identificar, valorar y adquirir conocimiento externo crítico para sus operaciones» (Forés \& Camisón, 2008, p. 37). Por su parte, la asimilación la abordan como la capacidad de la empresa para absorber el conocimiento externo, que puede ser operativo mediante las «rutinas y los procesos que le permiten a la empresa analizar, procesar, interpretar y comprender la información obtenida de fuentes externas» (Forés \& Camisón, 2008, p. 37). La transformación, es la habilidad para desarrollar y mejorar las rutinas internas que facilitan la transferencia y la combinación de los conocimientos previos con los ya 
adquiridos. Finalmente, la explotación la consideran como la competencia de la empresa para aplicar el nuevo conocimiento en los procesos organizacionales.

Lichtenthaler (2016b). Describe tres componentes principales: (a) Exploración del conocimiento, proceso mediante el cual se identifica y se adquiere el conocimiento útil externo mediante diferentes mecanismos de escaneo del entorno. En esta actividad, el dinamismo del entorno -cambios en los conocimientos, mercados y tecnología- tiene un efecto positivo sobre la orientación tecnológica y de mercado durante ese proceso de exploración (Lichtenthaler, 2016b), permitiendo a la empresa adecuarse a los últimos desarrollos en los distintos campos de acción. (b) Retención de conocimientos, se refiere al mantenimiento y reactivación del conocimiento en la organización a lo largo del tiempo. (c) Explotación del conocimiento, consiste en la transformación del conocimiento en nuevos productos y servicios. En resumen, Lichtenthaler (2016b) señala que esta orientación proactiva de la organización se constituye en un elemento motivador de los procesos organizacionales.

\section{Determinantes y medición de la capacidad de absorción}

La capacidad de absorción puede estar influenciada por diversas fuentes de conocimientos externo e internos (Van den Bosch et al., 1999; Todorova \& Durisin, 2007), tales como el conocimiento previo, acumulado por su experiencia, competencias básicas y el lenguaje común compartido, que facilitan y promueven el reconocimiento del valor de la información, su asimilación, transformación y aplicación (Todorova \& Durisin, 2007; Mariano \& Walter, 2015). Depende, además, de la formación de los miembros de la organización y de sus conocimientos acumulados (Roberts et al., 2012), de la inversión e intensidad en $\mathrm{I}+\mathrm{D}$, así como la existencia de unidades organizativas dedicadas a las actividades de investigación, al número de trabajadores con grados universitarios con alto perfil para la $\mathrm{I}+\mathrm{D}$, la cantidad de patentes y la proporción de personal científico y técnico en relación con el total de números de empleados (Escribano et al., 2009; Kostopoulos et al., 2011; Valentim et al., 2016). Todo lo cual se conjunta en distintas formas de medición y cuantificación de la CA, que han sido estudiadas en diversas investigaciones de carácter cualitativo y cuantitativo (Duchek, 2015; Valentim et al., 2016).

En la tabla 3, se describen brevemente algunos determinantes de la CA. 
Tabla 3. Trabajos sobre Determinantes de la Capacidad de Absorción (CA)

Autor

\begin{tabular}{|c|c|}
\hline $\begin{array}{l}\text { Van den } \\
\text { Bosch et al. } \\
\text { (1999) }\end{array}$ & $\begin{array}{l}\text { (1) Forma organizacional. La estructura funcional posee un alto potencial de } \\
\text { eficiencia debido a la economía de escala en el uso de los recursos, pero } \\
\text { baja flexibilidad en el proceso de absorción del conocimiento, puesto } \\
\text { que es más adaptada a entornos estables con mercados y productos de } \\
\text { ciclos de vida relativamente largos. La divisional, posee una mayor auto- } \\
\text { nomía en términos operacionales y mayor contacto directo con su en- } \\
\text { torno. Plantean que este tipo de estructura tiene un alto potencial para } \\
\text { la flexibilidad de la absorción del conocimiento por su mayor capaci- } \\
\text { dad para desenvolverse en entornos dinámicos. Y las formas matriciales, } \\
\text { tienen alta flexibilidad para la absorción del conocimiento por su ca- } \\
\text { pacidad de compartirlo entre las unidades que integran los proyectos. }\end{array}$ \\
\hline $\begin{array}{l}\text { Forés \& } \\
\text { Camisón } \\
(2008)\end{array}$ & $\begin{array}{l}\text { Determinantes internos y externos. Los internos: conocimientos de la em- } \\
\text { presa, cultura de innovación y aprendizaje, diseño organizativo abier- } \\
\text { to al aprendizaje, sistemas de gestión del conocimiento, edad y ta- } \\
\text { maño de la empresa. Los externos: grado de turbulencia del entorno, } \\
\text { existencia de oportunidades tecnológicas, características del conoci- } \\
\text { miento de otras empresas y distancia geográfica. }\end{array}$ \\
\hline \multirow{2}{*}{$\begin{array}{l}\text { Vega-Ju- } \\
\text { rado et al. } \\
(2008)\end{array}$} & $\begin{array}{l}\text { Conocimiento Organizacional: El conjunto de habilidades, experiencias, for- } \\
\text { mación e información que posee y ha acumulado la empresa producto } \\
\text { de los procesos de adquisición, aprendizaje, generación y aplicación. } \\
\text { Formalización: Aumenta la eficiencia en la adquisición de conocimientos } \\
\text { al establecer pautas de comportamiento; es decir, permite actuar con } \\
\text { baja supervisión, y facilita la creación de la memoria organizacional. }\end{array}$ \\
\hline & $\begin{array}{l}\text { Mecanismos de integración social: Formales e informales asociados a las } \\
\text { prácticas administrativas, tales como: rotación de personal, forma- } \\
\text { ción de equipos, círculos de calidad y metodologías para resolver pro- } \\
\text { blemas de participación e integración que facilitan la transferencia, la } \\
\text { transformación y la explotación del conocimiento. }\end{array}$ \\
\hline \multirow{3}{*}{$\begin{array}{l}\text { Schillaci et } \\
\text { al. (2013) }\end{array}$} & $\begin{array}{l}\text { Habilidades del Recurso Humano: Relacionado con su nivel de conocimien- } \\
\text { to, experiencia y formación, que facilita el acceso al conocimiento del } \\
\text { entorno. }\end{array}$ \\
\hline & $\begin{array}{l}\text { (2) Inversión } I+D \text { : Volumen de recursos financieros utilizados en la ad- } \\
\text { quisición de tecnología, conocimientos, equipos o cualquier otro re- } \\
\text { curso para ejecutar proyectos de investigación. }\end{array}$ \\
\hline & $\begin{array}{l}\text { (3) Estructura Organizativa: su adecuado diseño facilita la capacidad de la } \\
\text { empresa para organizar y estimular la transferencia de conocimien- } \\
\text { tos a través de todas las unidades y personas que forman parte de ella. }\end{array}$ \\
\hline
\end{tabular}




\begin{tabular}{|c|c|}
\hline Autor & Determinantes \\
\hline $\begin{array}{l}\text { Agui- } \\
\text { lar-Olaves et } \\
\text { al. (2014) }\end{array}$ & $\begin{array}{l}\text { Clasifican los determinantes según su capacidad de absorción potencial (PA- } \\
\text { CAP) y capacidad de absorción realizada (RACAP). En la primera, identi- } \\
\text { fican las fuentes externas de conocimientos: clientes, competidores, } \\
\text { consultoras, instituciones universitarias, centro de investigación y } \\
\text { congresos. Por otra parte, las actividades que estimulan RACAP: im- } \\
\text { plementación, aplicación, conversión y re-codificación del esfuerzo } \\
\text { innovador, los procesos tecnológicos, y la introducción de nuevos pro- } \\
\text { ductos o mejora de los existentes. }\end{array}$ \\
\hline $\begin{array}{l}\text { González } \\
\text { \& Hurtado } \\
(2014)\end{array}$ & $\begin{array}{l}\text { Los autores identificaron los siguientes indicadores: (a) Fase de adquisición: } \\
\text { Inversiones en I+D, en transferencia tecnológica y en ingeniería de di- } \\
\text { seño. (b) Fase asimilación: cooperación con proveedores e instituciones } \\
\text { para la realización de actividades de científicas, tecnológicas e inno- } \\
\text { vación. (c) Fase transformación: formación de los empleados, inversión } \\
\text { en asistencia técnica y consultoría. (d) Fase explotación: registro de pro- } \\
\text { piedad intelectual, mejora en la calidad de los productos o servicios, } \\
\text { o ampliación de su oferta. }\end{array}$ \\
\hline $\begin{array}{l}\text { Duchek } \\
(2015)\end{array}$ & $\begin{array}{l}\text { Las actitudes y preferencias de los actores en los procesos organi- } \\
\text { zativos son determinantes de la CA. Las desviaciones de las reglas } \\
\text { formales son una condición importante para la absorción flexible del } \\
\text { conocimiento y, por tanto, para altos niveles de CA. Significa que la } \\
\text { dirección debe aceptar que las desviaciones de las reglas pueden ser } \\
\text { necesarias y en algunos casos alentarse. }\end{array}$ \\
\hline $\begin{array}{l}\text { Lichtentha- } \\
\text { ler (2016b). }\end{array}$ & $\begin{array}{l}\text { La orientación tecnológica y proactiva de la empresa hacia el mer- } \\
\text { cado afecta positivamente los procesos de exploración, retención y } \\
\text { explotación de la CA. (2) El dinamismo del entorno tiene un efec- } \\
\text { to moderador positivo en la relación de la empresa con el grado de } \\
\text { orientación tecnológica y proactivo hacia el mercado, y en el nivel de } \\
\text { exploración, retención y explotación del conocimiento. }\end{array}$ \\
\hline $\begin{array}{l}\text { Zou et al. } \\
\text { (2018). }\end{array}$ & $\begin{array}{l}\text { Los autores realizan un meta-análisis sobre el concepto de capacidad de absor- } \\
\text { ción y ubican dos determinantes fundamentales: el tamaño y la edad de la em- } \\
\text { presa. De ese análisis, encuentran una relación positiva para las peque- } \\
\text { ñas empresas y negativa para las grandes. Por su parte, la relación es } \\
\text { negativa para las empresas maduras y no significativa en las jóvenes. }\end{array}$ \\
\hline
\end{tabular}

\section{La cognición del individuo y la capacidad absorción}

Desde una visión de la psicología cognitiva, Qian \& Jung (2017) argumentan que la capacidad absorción es un mecanismo efectivo para solventar los problemas de los filtros de información y de conocimientos durante el proceso de reconocimiento de su valor efectivo para la organización. Esos filtros pueden explicarse dentro del marco de la teoría cognitiva a través de los procesos, los sesgos y los mapas cognitivos que en su conjunto configuran la cognición del individuo y su capacidad cognitiva.

Los procesos cognitivos permiten entender cómo el conocimiento es seleccionado, organizado, transformado, almacenado y utilizado a través de la percepción, la 
interpretación, la atención, el pensamiento, la memoria y el lenguaje de los individuos (Neisser, 1981; Banyard et al., 1995). Por otra parte, los sesgos cognitivos constituyen una tendencia a pensar, a percibir o a recordar de una manera particular (Banyard et al., 1995), por lo que los individuos responsables de la toma de decisiones estratégicas dependen en buena medida de algunos juicios o sesgos cognitivos -predisposiciones mentales- para simplificar o reducir las tareas y decisiones en situaciones complejas (Zapata \& Canet, 2009) $)^{2}$.

Los mapas o esquemas cognitivos además de que representan un cúmulo de conocimientos, experiencias e imágenes del mundo, también sugieren una estructura activa para la búsqueda, depuración e interpretación de la información utilizada para dirigir y formar en la mente del individuo las percepciones del entorno que le rodea (Zapata \& Canet, 2009). Constituye así una estructura cognitiva compuesta de una red de expectativas aprendidas de la experiencia y almacenada en la memoria, y por tanto ayuda a las personas a simplificar, filtrar y manejar eficientemente la información, así como direccionar los cursos de acción bajo un contexto complejo y de incertidumbre (Gioia, 1986; Banyard et al., 1995; Hodgkinson et al., 2004).

Child (1997) sostiene que el ejercicio de la actividad estratégica y el diseño organizativo implican una evaluación previa del entorno por parte de quienes dirigen a la organización. Esa evaluación se lleva a cabo mediante filtros cognitivos que se dan durante la percepción (Milliken, 1987; Child, 1997; Walton y Dawson, 2001; Zabkar et al., 2013). De esta forma, Weick (1979) señala que las organizaciones sólo reconocen a su entorno mediante el «proceso de percepción» de quienes las dirigen, siendo producto de una ordenación abreviada, generalizada y corregible de las distintas experiencias dentro de la mente que sirven de marco de referencia inicial para la acción, transformados en mapas y sesgos cognitivos ${ }^{3}$ (Zapata et al., 2015), y que influyen luego en esas percepciones (Zapata \& Canet, 2009).

Es así, como la visión cognitiva y la voluntad de los agentes define en buena medida el entorno donde la empresa va a participar y competir, lo que configura la perspectiva voluntarista. Es posible entonces hablar de un determinismo cognitivo; es decir, la voluntad apresada por la propia visión y concepción de las cosas (Peris et al., 2006), que en esencia constituyen la visión directiva o gerencial vinculada con la forma cómo la empresa debe ser organizada, dirigida y planificada. Todo ello, contribuye en la formación de la capacidad de absorción de la organización.

En resumen, se puede formular la siguiente proposición general (PG):

PG: Los procesos, los mapas y los sesgos cognitivos, que constituyen la cognición del individuo, tienen un efecto positivo y significativo sobre la capacidad de absorción de la organización.

Finalmente, los factores determinantes de la capacidad de absorción de la organización pueden integrarse en: cognitivos, organizacionales y del entorno.

2 En el enfoque de control externo se ubican las teorías: contingente, dependencia de recursos, institucional y ecología de las poblaciones. Forman también parte del enfoque de adaptación.

3 En el marco de la psicología cognitiva, se han identificado una serie de sesgos cognitivos: tendencia conservadora, anclaje, disponibilidad, ilusión de control, percepción selectiva, ley de pequeños números, proximidad, entre otros. 
Figura 1. Factores Determinantes de la Capacidad de Absorción

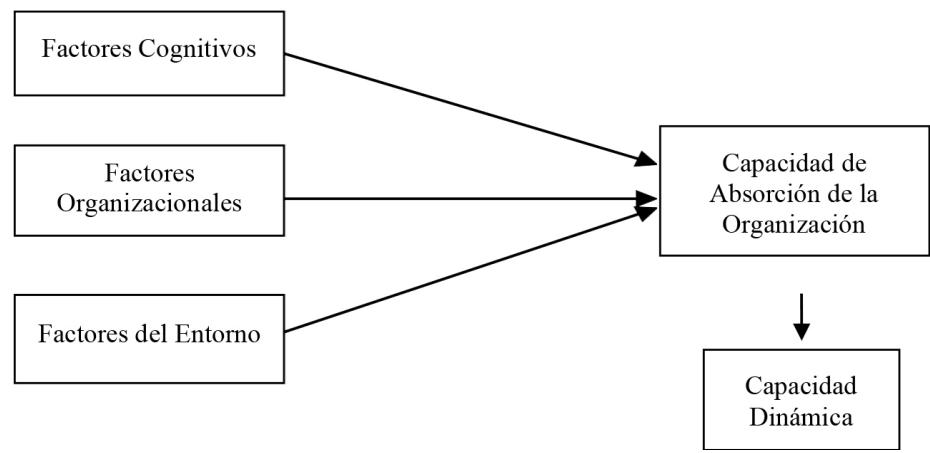

En la tabla 4 se ofrecen algunos de estos factores determinantes.

Tabla 4. Factores Determinantes de la Capacidad de Absorción

\begin{tabular}{|c|c|}
\hline $\begin{array}{l}\text { Factores } \\
\text { Cognitivos }\end{array}$ & $\begin{array}{l}\text { - Sesgos cognitivos. } \\
\text { - Mapas cognitivos. } \\
\text { - Procesos cognitivos. }\end{array}$ \\
\hline $\begin{array}{c}\text { Factores } \\
\text { Organizacionales }\end{array}$ & 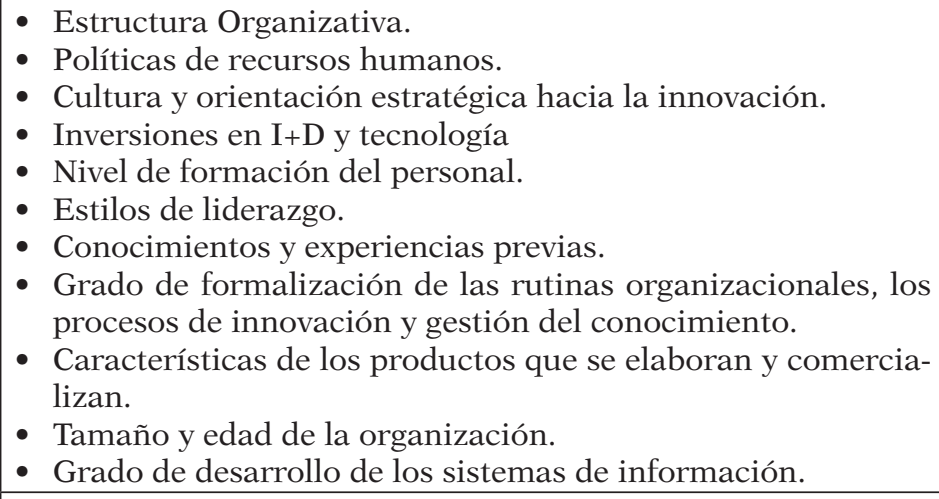 \\
\hline $\begin{array}{l}\text { Factores del } \\
\text { Entorno }\end{array}$ & $\begin{array}{l}\text { - Relaciones y acuerdo de cooperación con otras organizacio- } \\
\text { nes involucradas con los procesos de innovación y desarrollo } \\
\text { de conocimientos (universidades, institutos de formación e } \\
\text { investigación). } \\
\text { - Grado de dinamismo del entorno y prácticas organizaciona- } \\
\text { les (competencia, clientes, proveedores y asociados). } \\
\text { - Política gubernamental vinculada con la innovación y la ge- } \\
\text { neración de conocimientos. } \\
\text { - Conocimientos y tecnología disponible en los participantes } \\
\text { del entorno organizacional. } \\
\text { - Características de los productos y servicios que demanda el } \\
\text { entorno. }\end{array}$ \\
\hline
\end{tabular}




\section{A modo de conclusión}

Mediante una amplia revisión de la literatura especializada basada en investigaciones de carácter teórico y empírico, en este documento se ha hecho un intento por avanzar en el estudio y comprensión de la capacidad de absorción de la organización. A partir de su estudio y aplicación a través de sus diferentes componentes y formas de medición, se observa que se trata de un constructo que contribuye en buena medida a comprender el funcionamiento y comportamiento de la organización frente a un entorno cada vez más dinámico, complejo y competitivo, y sobre todo cuando se trata de los procesos de aprendizaje, innovación y gestión del conocimiento.

En la literatura se ha hecho un importante énfasis sobre la idea de que la CA mejora los resultados de innovación y los procesos de aprendizaje de la organización que conducen, por tanto, en la obtención de ventajas competitivas. Así, la CA concretada mediante la búsqueda, el reconocimiento y la adquisición del conocimiento externo para luego asimilarlo, integrarlo, transformarlo y aplicarlo en los procesos organizacionales se observa como una actividad fundamental para que la organización enfrente con éxito las exigencias y los cambios del entorno. Juega así un papel crucial en el aprendizaje y desempeño de la empresa, constituyéndose en una capacidad clave para sus procesos de innovación y competitividad (Oumaya \& Gharbi, 2017).

Es de destacar que aunque los estudios posteriores al trabajo seminal de Cohen \& Levinthal (1990) han conservado en esencia el espíritu inicial del concepto CA, se destaca que el cambio más importante fue el de Zahra \& George (2002) al asociarlo a un conjunto de rutinas y procesos estratégicos desarrollados a través de esfuerzos ordenados y persistentes con el fin de generar capacidades dinámicas (Forés \& Camisón, 2008; Camisón \& Forés, 2014). Este cambio viene acompañado por la propuesta que considera la CA dividida en dos sub-constructos no necesariamente vinculantes: «capacidad de absorción potencial» (PACAP) y «capacidad de absorción realizada» (RACAP). Surge a partir de esos cambios, la idea de que la CA es una capacidad dinámica de la organización, puesto que a través de ella la empresa puede alcanzar desempeños superiores y ventajas competitivas por medio de las actividades de búsqueda, adquisición, asimilación de conocimiento externo útil, y su transformación, transferencia y aplicación posterior en todas las actividades de la empresa. Lo resaltan Saiz et al. (2018, p. 290) al señalar que "la influencia de la capacidad de absorción potencial y realizada es significativa sobre los nuevos productos y causa efectos sobre la investigación y el desarrollo internos de diversas maneras».

Es así, que la CA es considerada como un componente de las capacidades dinámicas, convirtiéndose en un tema de estudio de primer orden tanto en la investigación académica como aplicada en el ámbito organizacional.

Un aporte importante de esta investigación es la propuesta de un modelo de los factores determinantes de la capacidad de absorción de la organización, establecidos en tres principales: cognitivos, organizativos y del entorno, que permiten de algún modo su mejor sistematización y comprensión.

Cabe destacar que la revisión de la literatura realizada, indica que aunque se ha avanzado en el estudio de la CA con respecto a sus componentes, determinantes y formas de medición; de igual modo se nota poco abordado y evolucionado el estudio 
sobre los efectos de la capacidad cognitiva de quienes dirigen a la organización, definida por los procesos, los mapas y los sesgos cognitivos cuyas bases conceptuales se encuentran en la psicología cognitiva, y que en este trabajo se han identificado como factores cognitivos. Lo anterior se considera una línea de investigación que promete hallazgos importantes para el entendimiento del comportamiento de las organizaciones y de quienes forman parte de ella, y de su vinculación con la capacidad de absorción y gestión del conocimiento.

\section{Apoyo y agradecimiento}

Este artículo se deriva del proyecto de investigación titulado: Las Capacidades Dinámicas de la Organización: Estudio Teórico y un Modelo Propuesto. Se llevó a cabo con el apoyo del Consejo de Desarrollo Científico Humanístico, Tecnológico (CDCHT) de la Universidad Centroccidental Lisandro Alvarado, Venezuela. Está identificado con el código de proyecto: 005-RAC-2015.

\section{Referencias}

Arias-Pérez, J., Coronado, J. \& Perdomo, G. (2017). Capacidad de absorción del rival y desempeño innovador: efecto mediador de la orientación estratégica. Revista Lasallista de Investigación, 14(2), 84-91. https://doi.org/10.22507/rli.v14n2a8.

Aguilar-Olaves, G., Herrera, L., \& Clemenza, C. (2014). Capacidad de absorción: aproximaciones teóricas y empíricas para el sector servicio. Revista Venezolana de Gerencia, 19(67), 499-518.

Banyard, P., Cassells, A., Green, P., Hartland, J., Hayes N., \& Reddy, P. (1995). Introducción a los Procesos Cognitivos. Ariel Psicología. Barcelona, España.

Camisón, C. \& Forés, B. (2014). Capacidad de absorción: antecedentes y resultados. Economía Industrial, 391, 13-22.

Cassol, A., Reis, C., \& Lima, R. (2016). Redefining the Relationship between Intellectual Capital and Innovation: The Mediating Role of Absorptive Capacity. Brazilian Administration Review, 13(4), 1-25. https://doi.org/10.1590/1807-7692bar2016150067

Cepeda I., Martelo, S., \& Leal, A.(2016). Absorptive Capacity and Value in the Banking Industry: A Multiple Mediation Model. Journal of Business Research, 69(5), 1644-1650. https://doi.org/ 10.1016/j.jbusres.2015.10.032.

Chang, Ch., Chen, Y., \& James, M. (2014). Determinants of Absorptive Capacity: Contrasting Manufacturing vs Services Enterprises. REDD Management, 44(5), 466-483. DOI: https://doi. org/10.1111/radm.12086

Charão, G. \& Matos, J. (2017). Absorptive Capacity: An Analysis in the Context of Brazilian Family Firms. Ram, Rev. Adm. Mackenzie, 18(1), 174-204. https://doi.org/10.1590/1678-69712017/ administracao.v18n1p174-204

Chen, Sh. \& Chang, B. (2012). The Effects of Absorptive Capacity and Decision Speed on Organizational Innovation: A Study of Organizational Structures an Antecedent Variable. Contemporary Management Research, 8(1), 27-50.

Child, J. (1997). Strategic Choice in the Analysis of Action, Structure, Organizations and Environment. Organization Studies, 18, 43-76.

Cohen, W., \& Levinthal, D. (1989). Innovation and Learning: The Two Faces of R\&D. Economic Journal, 99, 569-596.

Cohen, W., \& Levinthal, D. (1990). Absorptive Capacity: A New Perspective on Learning and Innovation. Administrative Science Quarterly, 35, 128-152. 
Cohen, W. \& Levinthal, D. (1994). Fortune Favors the Prepared Firm. Management Science, 40(2), 227-251.

Cruz, J., López, P., \& Martín de Castro, G. (2009). La influencia de las capacidades dinámicas sobre los resultados financieros de la empresa. Cuadernos de Estudios Empresariales, 19, 105-128.

Duchek, S. (2013). Capturing Absorptive Capacity: A critical Review and Future Prospects. Schmalenbach Business Review, 65, 312-329.

Duchek, S. (2015). Designing Absorptive Capacity? An Analysis of Knowledge Absorption Practices in German High-Tech Firms. International Journal of Innovation Management, 19(4), 1-22. https://doi.org/10.1142/S1363919615500449.

Díaz, J. (2012). Guía práctica de lectura crítica de artículos científicos originales en ciencias de la salud. España: Instituto Nacional de Gestión Sanitaria.

Escribano, A., Fosfuri, A., \& Tribób, J. (2009). Managing External Knowledge Flows: The Moderating Role of Absorptive Capacity. Research Policy, 38, 96-105. https://doi.org/10.1016/j. respol.2008.10.022

Forés, B., \& Camisón, C. (2008). La capacidad de absorción de conocimiento: factores determinantes internos y externos. Dirección y Organización, 36, 35-50.

Gao, Sh., Yeoh, W., Wong, S., \& Scheeper, R. (2017). A Literature Analysis of the Use of Absorptive Capacity Construct in IS Research. International Journal of Information Management, 37, 36-42. https://doi.org/10.1016/j.ijinfomgt.2016.11.001

Garzón, M. (2015). Modelo de capacidades dinámicas. Revista Dimensión Empresarial, 13(1), 111-131.

Gárces, J. \& Duque, E. (2007). Metodología para el análisis y la revisión crítica de artículos de investigación. Innovar, 17(29), 184-194.

Gioia, D. (1986). Symbols, Scripts, and Sense Making: Creating Meaning in the Organizational Experience. En Dennis Gioia (Coord.), The Thinking Organization. San Francisco: Jossey-Bass, Inc., Publishers Editorial

González, C. \& Hurtado, A. (2014). Propuesta de un Indicador de Capacidad de Absorción del Conocimiento (icac-col): evidencia empírica para el sector servicios en Colombia. Rev. fac.cienc.econ., 22(2), 29-46.

Guisao, S., Rincón, L., \& Arias-Pérez, J. (2017). Capacidad de Tecnologías de Información y Desempeño Organizacional: Efecto Mediador de la Capacidad de Absorción. Cuaderno de Administración, 30(55). http:// dx.doi.org/10.11144/Javeriana.cao30-55.ctido.

Hoang, H., \& Rothaermel, R. (2005). The Effect of General and Partner-Specific Alliance Experience on Joint R\&D Project Performance. Academy of Management Journal, 48, 332-345. https://doi.org/10.5465/AMJ.2005.16928417.

Hodgkinson, G., Maule, J., \& Bown, N. (2004). Causal Cognitive Mapping in the Organizational Strategy Field: A Comparison of Alternative Elicitation Procedures. Organizational Research Methods, 7(1), 3-26.

Juknevičienė, V. (2017). Regional Absorptive Capacity and Regional Disparities in Lithuania: Linkages, Evidences and Insights. Scientific Papers of the University of Pardubice. Series D, Faculty of Economics $\mathbb{E}$ Administration, 25(40), 71-82.

Kang, M., \& Lee, M. (2017). Absorptive Capacity, Knowledge Sharing, and Innovative Behaviour of R\&D Employees. Technology Analysis $\mathcal{E}$ Strategic Management, 29(2), 219-232. https://doi.or $\mathrm{g} / 10.1080 / 09537325.2016 .1211265$.

Kostopoulos, K., Papalexandris, A., Papachroni, I., \& Margarit, G. (2011). Absorptive Capacity, Innovation, and Financial Performance. Journal of Business Research, 64, 1335-1343. https:// doi.org/10.1016/j.jbusres.2010.12.005.

King, A., \& Lakhani, K. (2011). The Contingent Effect of Absorptive Capacity: An Open Innovation Analysis. Harvard Business School. Working Paper.

Lane, P., \& Lubatkin, M. (1998). Relative Absorptive Capacity and Interorganizational Learning. Strategic Management Journal, 19, 461-477. 
Lane, P., Salk, J., \& Lyles, M. (2001). Absorptive Capacity, Learning, and Performance in International Joint Ventures, Strategic Management Journal, 22, 1139-1161. https://doi.org/10.1002/ smj.206.

Lane, P., Koka, B., \& Pathak, S. (2006). The Reification of Absorptive Capacity: A Critical Review and Rejuvenation of the Construct. Academy of Management Review, 31(4), 833-863. https:// doi.org/10.5465/AMR.2006.22527456.

Leiva, J., Rodríguez, J., \& Monge, R. (2017). Efectos de la contratación de empleados de multinacionales en la capacidad de absorción en empresas locales. Contaduría y Administración, 62, 657-669.

Lichtenthaler, U. (2016a). Absorptive Capacity and Firm Performance: An Integrative Framework of Benefits and Downsides. Technology Analysis $\mathcal{E}$ Strategic Management, 28(6), 664-676. https:// doi.org/10.1080/09537325.2015.1131258.

Lichtenthaler, U. (2016b). Determinants of Absorptive Capacity: The Value of Technology and Market Orientation for External Knowledge Acquisition. Journal of Business $\mathcal{E}$ Industrial Marketing, 31(5), 600-610. https://doi.org/10.1108/JBIM-04-2015-0076.

Lin, H., Su, J., \& Higgins, A. (2016). How Dynamic Capabilities Affect Adoption of Management Innovations. Journal of Business Research, 69, 862-876. https://doi.org/10.1016/j.jbusres.2015.07.004.

Marabelli, M., \& Newell, S. (2014). Knowing, Power and Materiality: A Critical Review and Reconceptualization of Absorptive Capacity, International Journal of Management Reviews, 16(4), 479-499. https://doi.org/10.1111/ijmr.12031.

Mariano, S., \& Walter, Ch. (2015). The Construct of Absorptive Capacity in Knowledge Management and Intellectual Capital Research: Content and Text Analyses. Journal of Knowledge Management, 19(2), 372-400. https://doi.org/10.1108/JKM-08-2014-0342.

Martinkenaite, I., \& Breunig, K. (2016). The Emergence of Absorptive Capacity Through Micro-Macro Level Interactions. Journal of Business Research, 69(2), 700-708. DOI: 10.1016/j. jbusres.2015.08.020.

Miguélez, E. \& Moreno, R. (2015). Knowledge Flows and the Absorptive Capacity of Regions. Research Policy, 44, 833-848. https://doi.org/10.1016/j.respol.2015.01.016.

Minbaeva, D., Pedersen, T., Björkman, I., Fey, C., \& Park, H. (2003). MNC Knowledge Transfer, Subsidiary Absorptive Capacity and Knowledge Transfer. Journal of International Business Studies, 34(6), 586-599.

Minbaeva, D., Pedersen, T., Björkman, I., \& Fey, C. (2014). A Retrospective on: MNC Knowledge Transfer Subsidiary Absorptive Capacity, and HRM. Journal of International Business Studies, 45(1), 52-62. https://doi.org/10.1057/jibs.2013.56.

Milliken, F. (1987). Three Types of Uncertainty About the Environment: State, Effect and Response Uncertainty. Academy of Management Review, 12(1), 133-143. https://doi.org/10.5465/ AMR.1987.4306502.

Neisser, U. (1981). Procesos cognitivos y realidad: principio e implicaciones de la Psicología Cognitiva. Madrid: Editorial Marova.

Oumaya, S., \& Gharbi, L. (2017). Individual and Collective Cbsorptive Capacities of New External Knowledge: The Case of Tunisian Small and Medium-Sized Enterprises (SmEs). International Journal of Technology Management $\mathcal{E}$ Sustainable Development, 16(3), 209-227. https:// doi.org/10.1386/tmsd.16.3.209_1.

Qian, H., \& Jung, H. (2017). Solving the Knowledge Filter Puzzle: Absorptive Capacity, Entrepreneurship and Regional Development, Small Business Economics, 48(1), 99-114. https://doi. org/10.1007/s11187-016-9769-y.

Penrose, E. (1959). The Theory of the Growth of the Firm. New York: Wiley.

Peltokorpi, V. (2017). Absorptive Capacity in Foreign Subsidiaries:The Effects Ofanguage-Sensitive Recruitment, Language Training, and Interunit Knowledge Transfer. International Business Review, 26(1), 119-129. https://doi.org/10.1016/j.ibusrev.2016.05.010. 
Peris, F., Peris-Ortiz, M., \& Zapata, G. (2006). Complementariedad de Teorías en la Administración Eficiente del Trabajo: Un Análisis Micro-organizativo. Compendium, 9(17), 75-94.

Pfeffer, J., \& Salancik, G. (1978). The External Control of Organizations: A Resource Dependence Perspective. New York: Harper \& Row, Publishers.

Roberts, N., Galluch, P., Dinger, M., \& Grover, V. (2012). Absorptive Capacity and Information Systems Research: Review, Synthesis, and Directions for Future Research. MIS Quarterly, 36(2), 625-648.

Rodríguez, L., \& Da Cunha, C. (2018). Impacts of Big Data Analytics and Absorptive Capacity on Sustainable Supply Chain Innovation: a Conceptual Framework. Scientific Journal of Logistics, 14(2), 151-161. https://doi.org/10.17270/J.LOG.2018.267.

Saiz, L., Pérez-Miguel, D., \& Manzanedo-del Campo, M. (2018). The Knowledge Absorptive Capacity to Improve the Cooperation and Innovation in the Firm. Journal of Industrial Engineering and Management, 11(2), 290-307. https://doi.org/10.3926/jiem.2505.

Sánchez, B., \& Nélida, M, (2017). Aportes teóricos a la gestión organizacional: la evolución en la visión de la organización. Ciencias Administrativas, 5(10), 65-74.

Schillaci, C., Romano, M., \& Nicotra, M. (2013). Territory's Absorptive Capacity. ERJ, 3(1), 109 126. https://doi.org/10.1515/erj-2012-0001.

Schweisfurth, T., \& Raasch, Ch. (2018). Absorptive Capacity for Need Knowledge: Antecedents and Effects for Employee Innovativeness. Research Policy, 47(4), 687-699. https://doi.org/10.1016/j.respol.2018.01.017.

Valencia-Rodríguez, M. (2015). Capacidades dinámicas, innovación de producto y aprendizaje organizacional en Pymes del sector cárnico. Ingeniería Industrial, 36(3), 297-305.

Valentim, L., Veríssimo, J., \& Franco, M. (2016). Knowledge Management Practices and Absorptive Capacity in Small and Medium-Sized Enterprises: Is There Really a Linkage? RED Management, 46(4), 711-725. https://doi.org/10.1111/radm.12108.

Van den Bosch, F., Volberda, H., \& Boer, M. (1999). Co-Evolution of Firm Absorptive Capacity and Knowledge Environment: Organizational Forms and Combinative Capabilities. Organization Science, 10, 551-568.

Vargas, J., \& Muratalla, G. (2017). Dynamic Capabilities Analysis in Strategic Management of Learning and Knowledge Absorption. RACE, 16(1), 227-260. https://doi.org/10.18593/ race.v16i1.10997.

Wang, C., \& Ahmed, P. (2007). Dynamic Capabilities: A Review and Research Agenda. International Journal of Management Reviews, 9(1), 31-51.

Walton, E., \& Dawson, S. (2001). Managers' Perceptions of Criteria of Organizational Effectiveness. Journal of Management Studies, 38(2), 173-199.

Weick, K. (1979). Cognitive Processes in Organization. Research in Organizational Behavior, 1, 47-74.

Vega-Jurado, J., Gutiérrez-Gracia, A., \& Fernández-de-Lucio, I. (2008). Analyzing the Determinants of Firm's Absorptive Capacity: Beyond R\&D. REDD Management, 38(4), 392-405. https://doi.org/10.1111/j.1467-9310.2008.00525.x.

Verona, G., \& Ravasi, D. (2003). Unbundling Dynamic Capabilities: An Exploratory Study of Continuous Product Innovation. Industrial and Corporate Change, 12, 577-606.

Viniegra, L. (2001). La crítica y el conocimiento. La Revista de Investigación Clínica, 53(2), 181-192.

Volberda, H. W., Foss, N. J., \& Lyles, M. A. (2010). Absorbing the Concept of Absorptive Capacity: How to Realize its Potential in the Organization Field. Organization Science, 21(4), 931-951. https://doi.org/10.1287/orsc.1090.0503.

Teece, D., Pisano, G., \& Shuen, A. (1997). Dynamic Capabilities and Strategic Management. Strategic Management Journal, 18(7), 509-533.

Teece, D., \& Leih, S. (2016). Uncertainty, Innovation, and Dynamic Capabilities: An Introduction. California Management Review, 58(4), 5-11. https://doi.org/10.1525/cmr.2016.58.4.5.

Teece, D., \& Linden, G. (2017). Business Models, Value Capture, and the Digital Enterprise. Journal of Organization Design, 6(8), 1-14. https://doi.org/10.1186/s41469-017-0018-x, 
Todorova, G., \& Durisin, B. (2007). Absorptive Capacity: Valuing a Reconceptualization. Academy of Management Review, 32(3), 774-786. https://doi.org/10.5465/AMR.2007.25275513.

Zapata, G., \& Canet, M. (2009). La cognición del individuo: reflexiones sobre sus procesos e influencia en la organización. Revista Espacio Abierto: Cuaderno Venezolano de Sociología, 18(2), 46-62.

Zapata, G., \& Hernández, A. (2014). Origen de los recursos y ventajas competitivas de las organizaciones: reflexiones teóricas. Revista Venezolana de Gerencia, 19(68), 735-759.

Zapata, G., Mirabal, A., \& Canet, M. (2015). El entorno de la organización: un estudio de sus tipologías y su vinculación con la percepción directiva y el diseño organizativo. Revista Ciencia y Sociedad, 40(4), 785-822.

Zapata, G., \& Mirabal, A. (2018). Capacidades dinámicas de la organización: revisión de la literatura y un modelo propuesto. Revista Investigación Administrativa, 47(121).

Zabkar, V., Cater, T., Bajde, D., \& Cater, B. (2013). Environmental Strategy: a Typology of Companies Based on Managerial Perceptions of Customer`s Environmental Activeness and Deterrents. E+M Ekonomie a Management, 16(3), 57-74.

Zahra, Sh., \& George, G. (2002). Absorptive Capacity: A Review, Reconceptualization and Extension. Academy of Management Review, 27(2), 185-203. https://doi.org/10.5465/ AMR.2002.6587995.

Zou, T., Ertug, G., \& George, G. (2018). The Capacity to Innovate: A Meta Analysis of Absorptive Capacity. Innovation: Organization $\mathcal{E}$ Management, 20(2), 87-121. https://doi.org/10.1080/1447 9338.2018.1428105. 